DOI: $10.15593 / 2224-9982 / 2018.54 .02$

УДК 536.331: 536.212

\author{
Н.А. Госсман, С.В. Русаков
}

Пермский государственный национальный исследовательский университет, Пермь, Россия

\author{
ВЛИЯНИЕ ГРАНИЧНЫХ УСЛОВИЙ ТЕПЛООБМЕНА \\ НА РАСЧЕТНОЕ ЗНАЧЕНИЕ ТЕПЛОВОГО СОСТОЯНИЯ КОРПУСА \\ КОМПРЕССОРА АВИАЦИОННОГО ДВИГАТЕЛЯ
}

\begin{abstract}
Достоверное определение теплового состояния компрессора высокого давления авиационного двигателя позволяет с более высокой точностью определять напряженно-деформированное состояние как ротора, так и статора компрессора.

В рамках данной работы исследовалось влияние граничных условий теплообмена на расчетное тепловое состояние заднего корпуса осевого компрессора авиационного двигателя. Оценивалось влияние коэффициента теплоотдачи в зоне радиальных зазоров между лопатками ротора и корпусом, величины термической проводимости в контактах и учет теплообмена излучением внутри корпуса на тепловое состояние деталей корпуса при моделировании в программном комплексе ANSYS Mechanical.

Расчеты заднего корпуса проводились в осесимметричной постановке. Неосесимметричные элементы, такие как детали системы автоматического управления радиальными зазорами, учитывались как осесимметричные. В программном комплексе ANSYS проведены следующие расчеты: без учета теплообмена излучением внутри корпуса и с учетом излучения, с двумя значениями теплового сопротивления в контактах между деталями, с увеличенными и уменьшенными на 10 \% значениями коэффициента теплоотдачи со стороны проточной части компрессора.

Результаты расчетов показали необходимость обязательного моделирования теплообмена излучением внутри заднего корпуса компрессора двигателя.

Ключевые слова: тепловое состояние корпуса компрессора, коэфффициент теплоотдачи, теплообмен излучением, система автоматического управления радиальными зазорами, ANSYS.
\end{abstract}

\author{
N.A. Gossman, S.V. Rusakov
}

Perm State National Research University, Perm, Russian Federation

\title{
THE INFLUENCE OF BOUNDARY CONDITIONS OF HEAT EXCHANGE ON THE CALCULATING THERMAL CONDITION OF THE AIRCRAFT ENGINE COMPRESSOR CASE
}

\footnotetext{
A reliable determination of the thermal state of the high-pressure compressor of an aircraft engine allows for more accurate determination of the stress-strain state of both the rotor and the compressor stator.

Within the framework of this work, the influence of the boundary conditions of heat exchange on the design thermal state of the rear shell of the axial compressor of an aircraft engine was investigated. The effect of the coefficient of heat transfer in the zone of radial gaps between the rotor blades and the hull, the thermal resistance in the contacts and the allowance for the heat exchange in the inside of the housing for the thermal state of the housing parts during simulation in the ANSYS software package was estimated.

Calculations of the rear hull were carried out in an axisymmetric setting. Non-axisymmetric elements, such as parts of the automatic control system for radial gaps, were considered as axisymmetric. In the ANSYS software complex, the following calculations were made: without taking into account the heat exchange within the housing and taking into account the radiation, with two values of the thermal resistance in the contacts between the parts, with increased and decreased by $10 \%$ values of the heat transfer coefficient from the compressor side.

The results of calculations showed the necessity of compulsory modeling of heat exchange by radiation inside the rear casing of the engine compressor.

Keywords: thermal state of the compressor casing, heat transfer coefficient, heat exchange by radiation, automatic control system for radial gaps, ANSYS.
} 


\section{Введение}

Детали корпусов компрессора высокого давления авиационного двигателя изготавливаются из материалов с высокими теплопрочностными свойствами. Рабочий температурный диапазон материалов должен соответствовать температурным режимам работы двигателя. Поскольку свойства материалов деталей, а именно коэффициент линейного расширения и модуль упругости, являются функциями температуры [1], от точности и достоверности определения теплового состояния деталей корпуса компрессора зависит точность расчетов его напряженнодеформированного состояния.

Основная проблема в расчетном определении теплового состояния деталей заключается в правильном определении граничных условий теплообмена. В работах $[2,3]$ указывается, что основным источником погрешности выступают коэффициенты теплоотдачи.

В данной работе исследовано влияние не только возможной погрешности при определении коэффициента теплоотдачи на тепловое состояние заднего корпуса компрессора высокого давления (КВД), но и таких факторов, как учет теплообмена излучением внутри корпуса и влияние погрешности определения величины теплового сопротивления в контактах.

\section{Расчетная модель}

\section{Основные уравнения}

Уравнение теплопроводности для стационарного случая в прямоугольных координатах $(x, y, z)$, в которых коэффициент теплопроводности $k=$ const, можно записать в виде [4]

$$
k_{x} \frac{\partial^{2} T}{\partial x^{2}}+k_{y} \frac{\partial^{2} T}{\partial y^{2}}+k_{z} \frac{\partial^{2} T}{\partial z^{2}}+q^{\prime \prime \prime}=0
$$

Система уравнений для конечных элементов будет иметь вид

$$
[\mathrm{K}]\{\mathrm{T}\}=\{\mathrm{Q}\},
$$

где $[\mathrm{K}]=$ матрица теплопроводности; $\{\mathrm{T}\}=$ вектор узловых температур; $\{\mathrm{Q}\}=$ вектор узловых тепловых потоков.

Внутри и снаружи рассматриваемого корпуса конвективный теплообмен происходит за счет вынужденного течения воздуха.

Граничное условие конвекции определяется следующим образом [5]:

$$
Q_{n}=\alpha\left(T-T_{\mathrm{B}}\right),
$$

где $Q_{n}$ - тепловой поток на границе деталей; $\alpha$ - коэффициент теплоотдачи; $T_{\text {в }}$ - температура воздуха; $T$ - температура деталей.

Внутри корпуса происходит свободный конвективный теплообмен, который возникает за счет разницы температур и также определяется уравнением (1). В данной работе свободный конвективный теплообмен внутри корпуса не учитывался.

Внутри корпуса также учитывается теплообмен излучением. Формула для расчета теплообмена между двумя непрозрачными телами на основании закона Стефана-Больцмана имеет вид [6]

$$
Q=\varepsilon_{\text {пр }} \cdot \sigma \cdot \varphi\left[\left(\frac{T_{1}}{100}\right)^{4}-\left(\frac{T_{2}}{100}\right)^{4}\right] \cdot F,
$$

где $Q$ - количество тепла, переданное излучением от первого тела ко второму; $\sigma$ - постоянная Стефана-Больцмана, $\sigma=5,67 \cdot 10^{-8} \mathrm{BT} /\left(\mathrm{m}^{2} \cdot \mathrm{K}^{4}\right) ; \varepsilon_{\text {пр }}$ - приведенная степень черноты системы; $\varphi-$ средний угловой коэффициент, учитывающий форму, размеры и взаимное расположение по- 
верхностей, $T_{1}$ и $T_{2}$ - абсолютные температуры первого и второго тела, К; $F$ - поверхность теплообмена, м².

Тепловая проводимость контакта между деталями определяется следующим соотношением [7]:

$$
\alpha_{\mathrm{\kappa}}=\frac{q}{\Delta T},
$$

где $q$ - плотность теплового потока; $\Delta T$ - скачок температур в зоне контакта.

\section{Граничные условия конвективного теплообмена к корпусу}

На рис. 1 показана расчетная осесимметричная модель корпуса в ANSYS с регионами задания граничных условий теплообмена.

Для построения КЭМ элементов корпуса используются элементы типа PLANE 55. Для моделирования контактов между деталями корпуса - пара элементов TARGE169 и CONTA172.

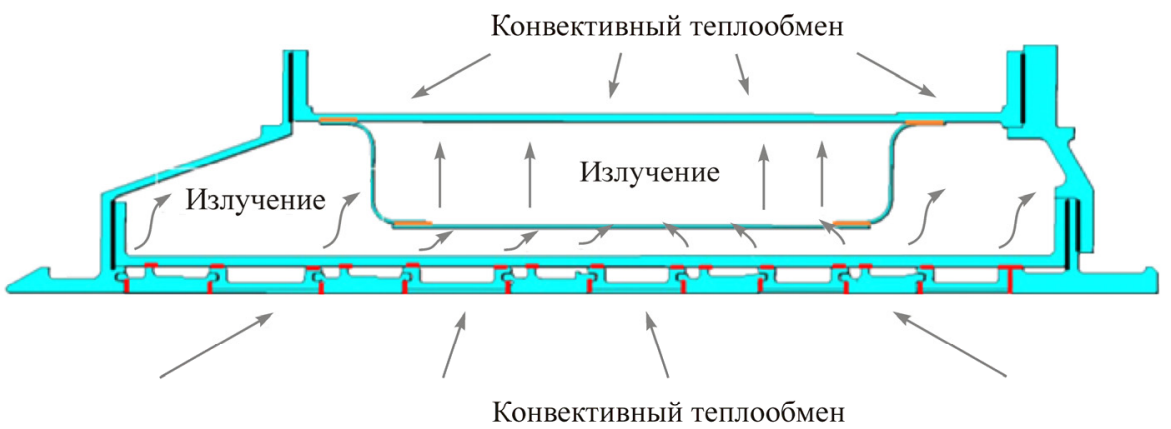

Рис. 1. Расчетная тепловая модель заднего корпуса компрессора высокого давления; зоны контактов выделены линиями: черная - болтовое соединение, желтая - соединение-сварка, красная - соединение с проскальзыванием

В качестве граничных условий конвективного теплообмена к корпусу задаются коэффициенты теплоотдачи и температура воздуха, описанные в уравнении (1).

Для определения коэффициента теплоотдачи в зоне радиального зазора рассматривалась эмпирическая зависимость, представленная в [8]:

$$
\alpha=\frac{\mathrm{Nu} \cdot \lambda_{\mathrm{cp}}}{b_{\mathrm{PK}}},
$$

где $\mathrm{Nu}=0,058 \cdot \operatorname{Re}^{0,8}\left(1-\bar{\Delta}_{Z}^{0,5}\right), \operatorname{Re}=\frac{c_{\mathrm{cp}} \cdot \rho_{\mathrm{cp}} \cdot b_{\mathrm{PK}}}{\mu_{\mathrm{cp}}}, \bar{\Delta}_{Z}=\frac{\Delta_{Z}}{h_{\mathrm{PK}}}-$ относительный радиальный зазор, где $\Delta_{Z}$ - радиальный зазор; $h_{\mathrm{PK}}$ - высота рабочей лопатки; $b_{\mathrm{PK}}-$ хорда рабочей лопатки в периферийном сечении; $\rho_{\text {ср }}$ - средняя плотность воздуха между входом и выходом из рабочего колеса в периферийном сечении; $c_{\text {ср }}$ - средняя абсолютная скорость между входом и выходом из рабочего колеса в периферийном сечении. Динамическая вязкость $\mu_{\text {ср }}$ определялась как функция температуры

$$
\mu_{\mathrm{cp}}=1,71 \cdot 10^{-5}\left(\frac{T_{\mathrm{cp}}}{273}\right)^{0,683}, \mathrm{H} \cdot \mathrm{c} / \mathrm{M}^{2}
$$


Значения скорости, давления и температуры (для определения плотности $\rho_{\text {ср }}$ ) воздуха в каждом рабочем колесе брались из результатов расчета аэродинамических параметров компрессора.

Коэффициент теплоотдачи корпуса на торцевых поверхностях направляющих лопаток определялся зависимостью

$$
\alpha=\frac{\mathrm{Nu} \cdot \lambda_{\mathrm{cp}}}{b_{\mathrm{HA}}},
$$

где $\mathrm{Nu}=0,032 \cdot \mathrm{Re}^{0,8}-$ число Нуссельта; $\mathrm{Re}=\frac{c_{\mathrm{cp}} \cdot \rho_{\mathrm{cp}} \cdot b_{\text {НА }}}{\mu_{\mathrm{cp}}}-$ число Рейнольдса; $\rho_{\mathrm{cp}}-$ средняя плотность воздуха между входом и выходом из направляющего аппарат; $\mu_{\mathrm{cp}}-$ средний динамический коэффициент вязкости между входом и выходом из направляющего аппарата; $\lambda_{\text {cp }}$ средний коэффициент теплопроводности между входом и выходом из направляющего аппарата; $c_{\mathrm{cp}}$ - средняя абсолютная скорость между входом и выходом из направляющего аппарата; $b_{\mathrm{CA}}$ - хорда направляющего аппарата на наружном диаметре. При использовании данной зависимости за определяющие параметры принимают средние параметры потока на входе и выходе из направляющего аппарата в абсолютном движении.

Коэффициент теплоотдачи снаружи корпуса определялся уравнением для кольцевого канала [9].

Тепловая проводимость контакта между деталями, определяемая соотношением (3), в случае болтового соединения принималась равной $5600 \mathrm{BT} /\left(\mathrm{M}^{2} \mathrm{~K}\right)$, для соединения «сварка» $10^{6} \mathrm{BT} /\left(\mathrm{м}^{2} \cdot \mathrm{K}\right)$, для подвижного соединения (с проскальзыванием) $-2800 \mathrm{BT} /\left(\mathrm{м}^{2} \cdot \mathrm{K}\right)$.

\section{Влияние учета теплообмена излучением}

Как правило, при определении теплового состояния корпусов турбин высокого давления авиационных двигателей учитывают теплообмен излучением между деталями корпуса [10]. При определении теплового состояния корпусов компрессоров данным фактором пренебрегают в связи с более низкими температурами по сравнению с турбиной высокого давления.

В данном разделе будет показано, насколько изменится расчетная температура в характерных местах корпуса компрессора в случае учета теплообмена излучением.

Учет теплообмена излучением внутри корпуса компрессора с системой автоматического управления радиальными зазорами (САУР3) [11] проводился конечными элементами [12] с использованием многопроцессорного пакета прикладных программ ANSYS. Расчеты выполнялись в стационарной постановке.

В программном комплексе ANSYS существует несколько способов учета излучения [13]:

- одномерным элементом лучистой связи LINK31;

- элементами с поверхностным эффектом SURF152;

- методом матрицы излучения;

- методом, использующий алгоритм диффузного отражения.

В данной задаче учет излучения теплообмена производился методом матрицы излучения. Данный метод используется для более сложных задач излучения, включающих две или более излучающих поверхностей. В данном методе генерируется матрица эффективных проводимостей. Входящие в матрицу эффективные проводимости включают как угловые коэффициенты поверхностных элементов, так и степени черноты поверхностей. После этого матрица используется в тепловом анализе в качестве суперэлемента MATRIX50.

Степень черноты, входящая в уравнение (2), зависит от способа обработки металла, его температуры и от многих других факторов. Зачастую определить данную величину можно только экспериментальным путем. В нижеприведенных расчетах степень черноты принималась постоянной и равной 0,6 . 
Для оценки влияния излучения было выполнено два расчета: без учета теплообмена излучением и с учетом теплообмена излучением. На рис. 2, 3 представлены результаты стационарных расчетов в характерных местах заднего корпуса на одном из режимов работы двигателя [11].

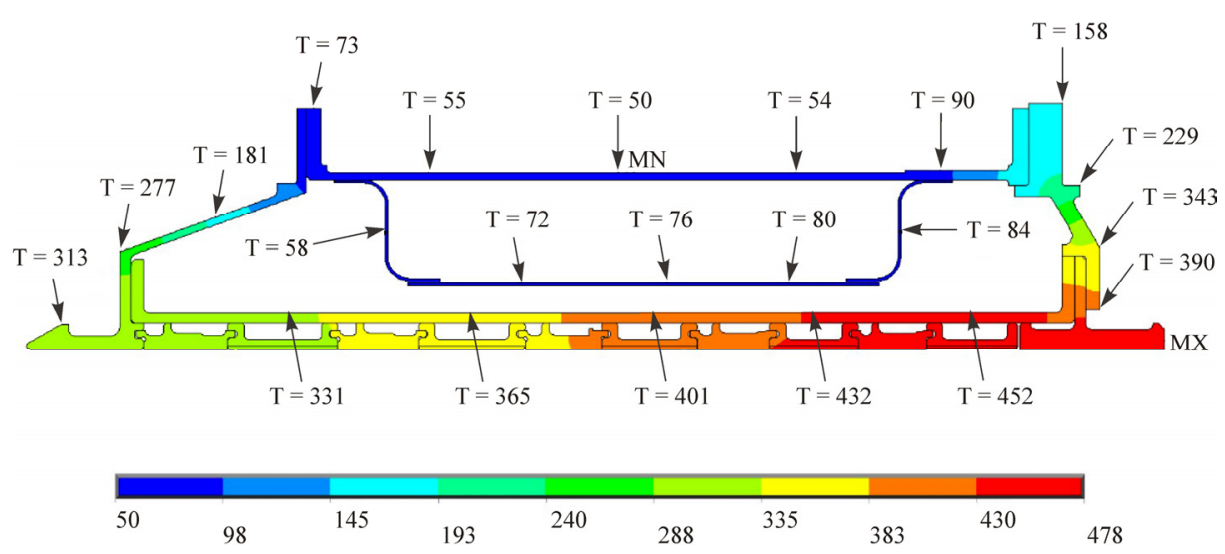

Рис. 2. Расчетное тепловое состояние заднего корпуса компрессора высокого давления без учета теплообмена излучением, ${ }^{\circ} \mathrm{C}$

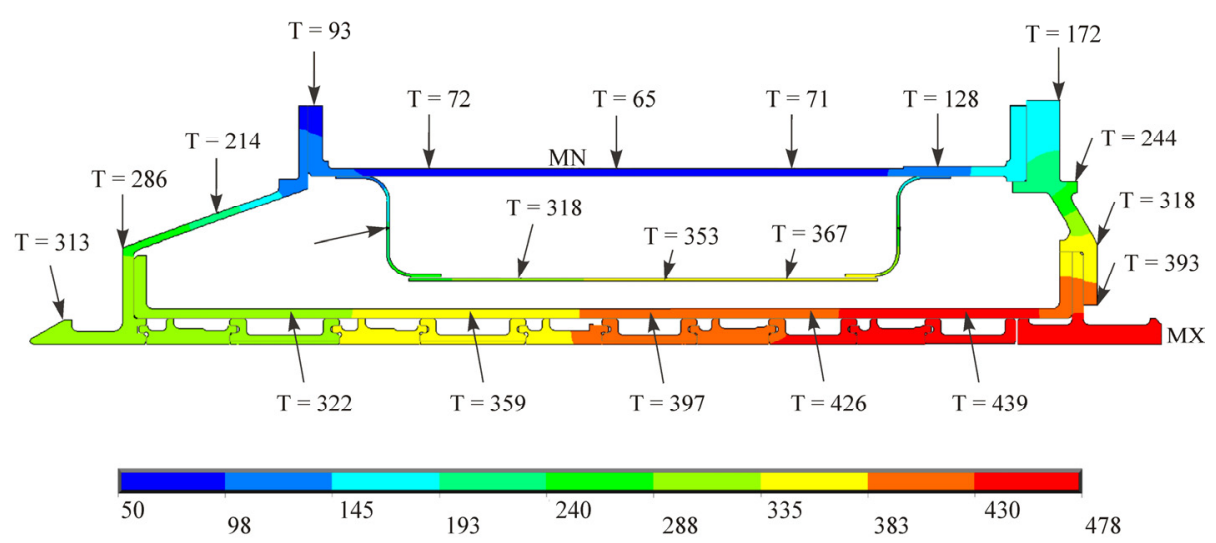

Рис. 3. Расчетное тепловое состояние заднего корпуса компрессора высокого давления с учетом теплообмена излучением, ${ }^{\circ} \mathrm{C}$

На рис. 4 представлена абсолютная разница между температурами в узлах КЭМ между расчетами с учетом теплообмена излучением и без учета.
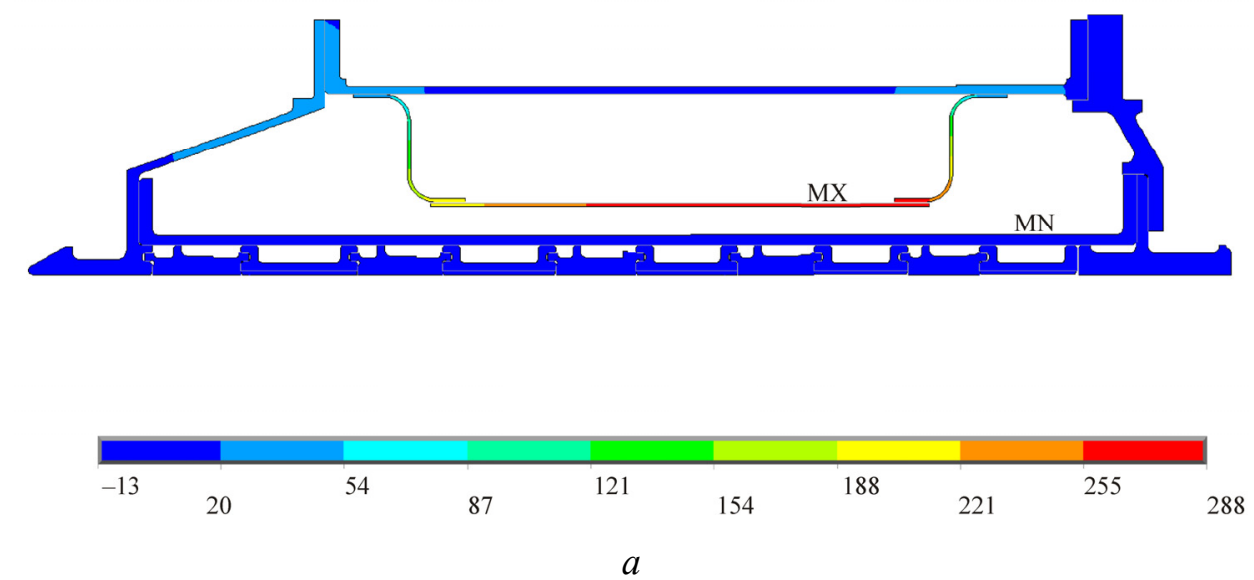

Рис. 4. Разница температур в узлах КЭМ заднего корпуса компрессора высокого давления между расчетами с учетом теплообмена излучением и без учета теплообмена излучением, ${ }^{\circ} \mathrm{C}$ (см. также с. 22 ) 

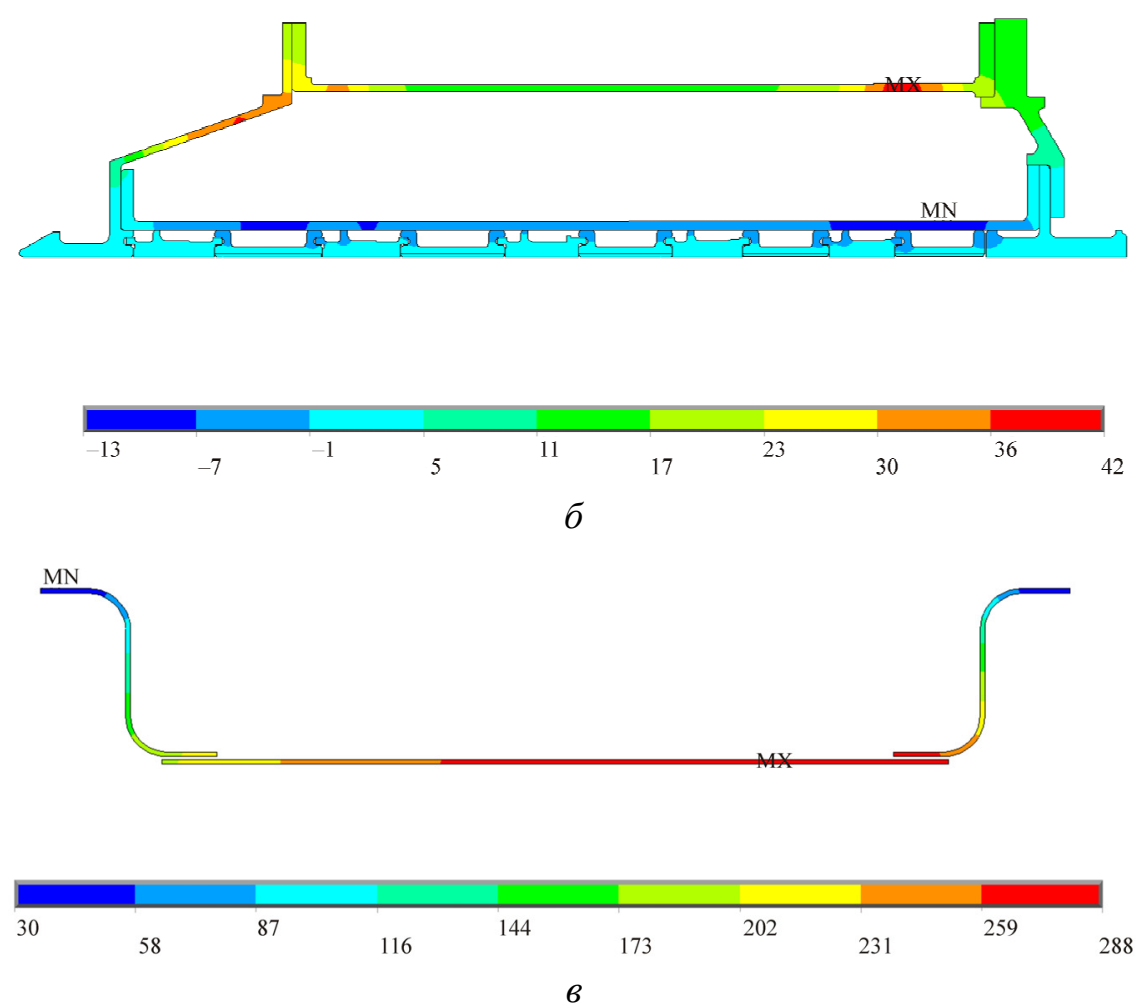

Рис. 4. Окончание

Как видно на рисунках, учет теплового излучения в тепловой расчетной модели существенно влияет на тепловое состояние деталей заднего корпуса компрессора, особенно детали САУРЗ (рис. 4, в). Также не значительно, но подогреваются фланцы корпуса, что может приводить к еще большему расширению всего корпуса на рассмотренном режиме работы двигателя.

\section{Влияние величины теплового контактного сопротивления}

В расчетной модели тепловые контакты моделируются с помощью элементов TARGE169 и CONTA172. В параметрах данных элементов необходимо задавать значение Thermal Contact Conductance (TCC), определяемое уравнением (3). В работах $[7,14]$ приводится подробное теоретическое описание, каким образом определять данную величину: с учетом шероховатости деталей, давления в контакте и т.п. На практике учитывать все эти факторы при определении теплового контактного сопротивления достаточно трудоемко. Поэтому данную величину определяют экспертно, на основании уже имеющихся экспериментальных данных.

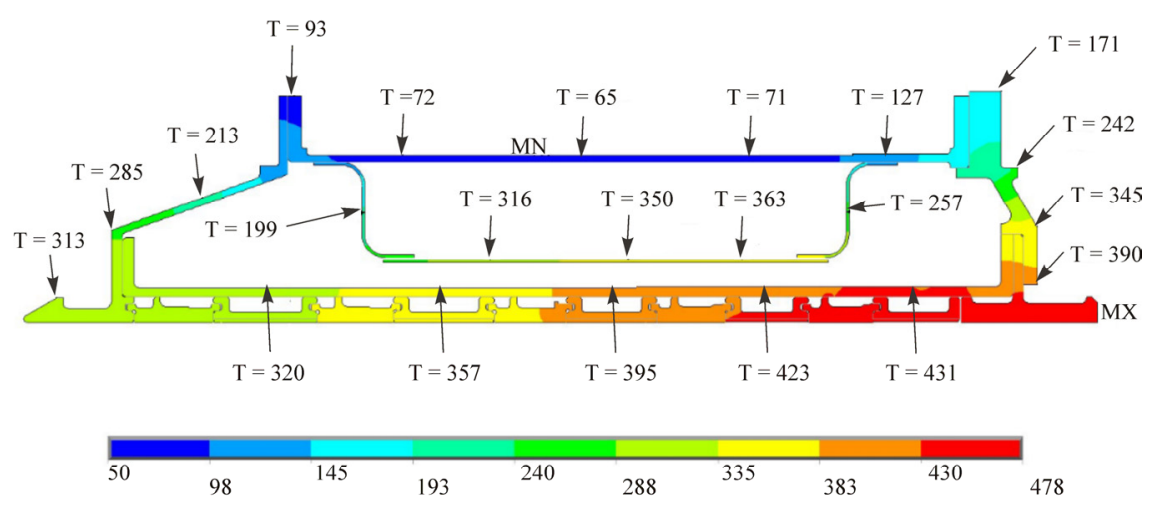

Рис. 5. Расчетное тепловое состояние заднего корпуса компрессора высокого давления с учетом излучения и ТСС $=1400 \mathrm{BT} / \mathrm{M}^{2} \cdot$ град, ${ }^{\circ} \mathrm{C}$ 
Для того чтобы определить, насколько сильно влияет погрешность при определении TCC, были выполнены расчеты с учетом теплообмена излучением с двумя значениям теплового контакта в соединениях с проскальзыванием (см. рис. 1, выделено красным): 2800 (Вт/м².град) (см. рис. 3) и 1400 (Вт/м².град) (рис. 5).

Как видно на рис. 3, 5, уменьшение теплового контактного сопротивления в два раза незначительно уменьшает температуру деталей внутреннего корпуса и деталей САУРЗ (максимальное отклонение составляет $\left.8{ }^{\circ} \mathrm{C}\right)$.

\section{Влияние коэффициента теплоотдачи в зоне радиальных зазоров и торцевых поверхностей}

Зависимости (4) и (5), описанные выше, позволяют определить коэффициенты теплоотдачи к внутренней части корпуса (проточной части). В работе [15] представлено расчетное влияние величины радиального зазора на значение коэффициента теплоотдачи в уравнении (4). В рамках данного исследования были выполнены тепловые расчеты при трех значениях коэффициентов теплоотдачи: определяемых уравнениями (4) и (5), уменьшенных на $10 \%$, увеличенных на $10 \%$.

Значения коэффициентов теплоотдачи, принятые в расчетах, представлены в таблице. Расположение регионов показано на рис. 6.

Значение коэффициентов теплоотдачи (исходные, увеличенные и уменьшенные на $10 \%), \mathrm{BT} / \mathrm{m}^{2}$ гррад

\begin{tabular}{|c|c|c|c|}
\hline Регион & Исходный & $-10 \%$ & $+10 \%$ \\
\hline NA8 & 4044 & 3640 & 4448 \\
\hline NA9 & 4417 & 3975 & 4858 \\
\hline NA10 & 4909 & 4418 & 5400 \\
\hline NA11 & 5341 & 4807 & 5875 \\
\hline NA12 & 5656 & 5090 & 6221 \\
\hline NA13 & 6000 & 5400 & 6600 \\
\hline RK9 & 7062 & 6356 & 7768 \\
\hline RK10 & 7660 & 6894 & 8426 \\
\hline RK11 & 8401 & 7561 & 9241 \\
\hline RK12 & 8956 & 8060 & 9851 \\
\hline RK13 & 9188 & 8269 & 10106 \\
\hline
\end{tabular}

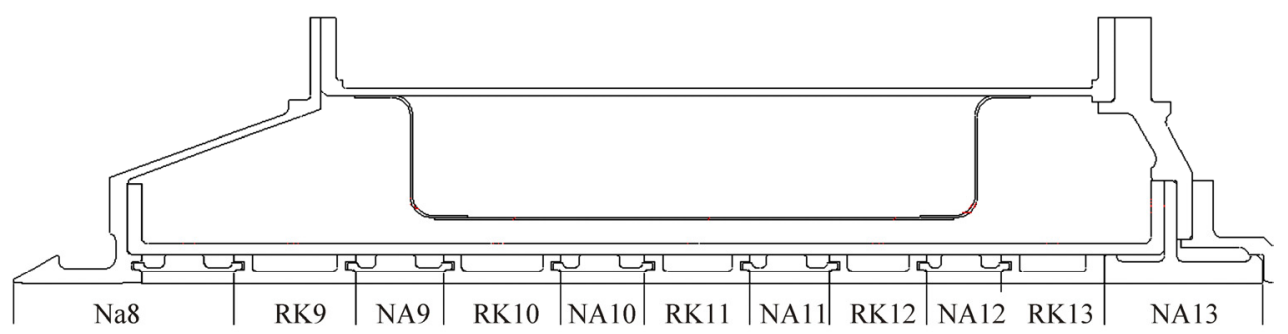

Рис. 6. Регионы приложения граничных условий с внутренней стороны заднего корпуса

Результаты расчетов показали, что в стационарной постановке уменьшение и увеличение значений коэффициентов теплоотдачи на $10 \%$ слабо влияют на тепловое состояние заднего корпуса компрессора (максимальное отклонение составляет $2{ }^{\circ} \mathrm{C}$ ). 


\section{Заключение}

Расчетная оценка влияния рассмотренных факторов на тепловое состояние заднего корпуса компрессора авиационного двигателя позволяет сделать вывод о необходимости обязательного учета теплообмена излучением между деталями корпуса. Необходима верификация результатов расчетов по экспериментальным данным термометрирования корпуса.

\section{Библиографический список}

1. Зиновьев В.Е. Теплофизические свойства металлов при высоких температурах. - М.: Металлургия, 1989. - 384 с.

2. Марценюк Е.В., Зеленый Ю.А., Климик Р.Р. Идентификация теплового состояния корпуса турбины авиационного двигателя по экспериментальным данным // Авиационно-космическая техника и технология. - 2016. - № 7(134). - С. 54-61.

3. Шиплюк А.Н. Методы оптимизация в задачах аэрогазодинамики. - Новосибирск: Изд-во НГТУ. - 2014. - 107 c.

4. Исаченко В.П., Осипова В.А., Сукомел А.С. Теплопередача: учебник для вузов. - Изд. 3-е, перераб. и доп. - М.: Энергия, 1975. -488 с.

5. Решение задач теплопроводности методом конечных элементов: учеб. пособие / Н.П. Жуков, Н.Ф. Майникова, С.С. Никулин, О.А. Антонов. - Тамбов: Изд-во ТГТУ, 2014. - 80 с.

6. Блох А.Г., Журавлев Ю.А., Рыжов Л.Н, Теплообмен излучением: справочник. - М.: Энергоатомиздат, 1991. $-432 \mathrm{c}$.

7. Попов В.М. Теплообмен в зоне контакта разъемных и неразъемных соединений. - М.: Энергия, 1971. $-216 \mathrm{c}$.

8. Теплопередача в охлаждаемых деталях газотурбинных двигателях / В.И. Локай, М.Н. Бодунов, В.В. Жуйков, А.В. Щукин. - 2-е изд., перераб. и доп. - М.: Машиностроение, 1993. - 288 с.

9. Швец И.Т., Дыбан Е.П. Воздушное охлаждение деталей газовых турбин. - Киев: Наукова думка, 1974. - 487 с.

10. Экспериментальное исследование теплового состояния корпусов газотурбинных двигателей / А.А. Григорьев, М.Ш. Нихамкин, Л.В. Воронов, Н.А. Саженков, А.Л. Полянин // Вестник Пермского национального исследовательского политехнического университета. Аэрокосмическая техника. - 2014. № 37. - С. 86-98.

11. Акуленко В.С., Иноземцев А.А., Соловьев Б.А. Авиационный газотурбинный двигатель ПС-90А: учеб. пособие. - Л., 1990.

12. Зенкевич О. Метод конечных элементов в технике. - М.: Мир, 1975.

13. Теплообмен излучением в ANSYS Mechanical [Электронный pecypc]. - URL: caeclub.ru/sites/default/files/teploobmen_izlucheniem_0.pdf.

14. Резник С.В., Просунцов П.В., Сапронов Д.В. Исследование теплового и механического контакта керамической лопатки и металлического диска в рабочем колесе газовой турбины // Теплофизические основы энергетических технологий: сб. ст. V Bсерос. науч. конф. с междунар. участием. Томск 15-17 октября 2014 г. - С. 325-336.

15. Госсман Н.А. Расчет влияния радиального зазора на граничные условия теплообмена к корпусу КВД авиационного двигателя // Неравновесные процессы в сплошных средах: материалы международного симпозиума. - Пермь, 2017. - С. 137-139.

\section{References}

1. Zinovyev V.E. Teplofizicheskiye svoystva metallov pri vysokikh temperaturakh [Thermophysical properties of metals at high temperatures. Reference edition]. Moscow: Metallurgiya, 1989, $384 \mathrm{p}$.

2. Martsenyuk E.V., Zelenyy Yu.A., Klimik R.R. Identifikatsiya teplovogo sostoyaniya korpusa turbiny aviatsionnogo dvigatelya po eksperimentalnym dannym [Identification of the thermal state of the turbine housing of an aircraft engine based on experimental data]. Aerospace technic and technology, 2016, No. 7 (134), pp. 54-61.

3. Shiplyuk A.N. Metody optimizatsiya $v$ zadachakh aerogazodinamiki [Methods of optimization in aerogasdynamic problems]. Novosibirsk: Publishing house of the NSTU, 2014, 107 p.

4. Isachenko V.P., Osipova V.A., Sukomel A.S. Teploperedacha. Uchebnik dlya vuzov [Heat transfer. Textbook for high schools, ed. 3rd, revised. and additional]. Moscow: Energiya, 1975, 488 p. 
5. ZHukov N.P., Maynikova N.F., Nikulin S.S., Antonov O.A. Resheniye zadach teploprovodnosti metodom konechnykh elementov: uchebnoye posobiye [Solving the problems of heat conduction by the finite element method: a textbook]. Tambov: Publishing House of the Federal State Educational Establishment of Higher Professional Education "TSTU", 2014, 80 p.

6. Blokh A.G., ZHuravlev Yu.A., Ryzhov L.H, Teploobmen izlucheniyem: Spravochnik [Heat exchange by radiation: Handbook]. Mosccow: Energoatomizdat, 1991, 432 p.

7. Popov V.M. Teploobmen v zone kontakta razyemnykh i nerazyemnykh soyedineniy [Heat transfer in the contact zone of detachable and non-detachable connections]. Moscow: Energiya, 1971, 216 p.

8. Lokay V.I., Bodunov M.N., Zhuykov V.V., Shchukin A.V. Teploperedacha v okhlazhdayemykh detalyakh gazoturbinnykh dvigatelyakh [Heat transfer in cooled parts of gas turbine engines. 2 nd ed., Revised. and additional]. Moscow: Mashinostroyeniye, 1993, 288 p.

9. Shvets I.T., Dyban E.P. Vozdushnoye okhlazhdeniye detaley gazovykh turbin [Air cooling of gas turbine parts]. Kiev: Naukova Dumka, 1974, 487p.

10. Grigoryev A.A., Nikhamkin M.SH., Voronov L.V., Sazhenkov N.A., Polyanin A.L. Eksperimentalnoye issledovaniye teplovogo sostoyaniya korpusov gazoturbinnykh dvigateley [Experimental study of the thermal state of gas turbine engine bodies]. PNRPU Aerospace Engineering Bulletin, 2014, no. 37, pp. 86-98.

11. Akulenko V.S., Inozemtsev A.A., Solovyev B.A. Aviatsionnyy gazoturbinnyy dvigatel PS-90A [Aviation gas-turbine engine PS-90A, educational allowance]. Leningrad, 1990.

12. Zenkevich O. Metod konechnykh elementov v tekhnike [Finite Element Method in Engineering]. Moscow: Mir, 1975.

13. Teploobmen izlucheniyem v ANSYS Mechanical [Heat exchange by radiation in ANSYS Mechanical]. URL: cae-club.ru/sites/default/files/teploobmen_izlucheniem_0.pdf

14. Reznik S.V., Prosuntsov P.V., Sapronov D.V. Issledovaniye teplovogo i mekhanicheskogo kontakta keramicheskoy lopatki i metallicheskogo diska $\mathrm{v}$ rabochem kolese gazovoy turbiny [Investigation of the thermal and mechanical contact of a ceramic blade and a metal disk in the impeller of a gas turbine]. Thermophysical fundamentals of energy technologies. Collection of articles of the V All-Russian Scientific Conference with international participation. Tomsk, October 15-17, 2014, pp. 325-336.

15. Gossman N.A. Raschet vliyaniya radialnogo zazora na granichnyye usloviya teploobmena k korpusu KVD aviatsionnogo dvigatelya [Calculation of the influence of the radial clearance on the boundary conditions of heat exchange to the aircraft engine air-pressure vessel]. Nonequilibrium processes in continuous media. Materials of the international symposium, 2017, pp. 137-139.

\section{Об авторах}

Госсман Наталья Александровна (Пермь, Россия) - аспирант кафедры «Прикладная математика и информатика» ФГБОУ ВО ПНИПУ (614990, г. Пермь, ул. Букирева, д. 15, e-mail: ngossman@ mail.ru).

Русаков Сергей Владимирович (Пермь, Россия) - доктор физико-математических наук, професcop, заведующий кафедрой «Прикладная математика и информатика» ФГБОУ ВО ПНИПУ (614990, г. Пермь, ул. Букирева, д. 15, e-mail: rusakov@psu.ru).

\section{About the authors}

Natalia A. Gossman (Perm, Russian Federation) - PhD Student, Department of Applied Mathematics and Informatics, Perm State National Research University (15, Bukirev st., Perm, 614990, Russian Federation, e-mail: ngossman@mail.ru).

Sergey V. Rusakov (Perm, Russian Federation) - Doctor of Physical and Mathematical Sciences, Professor, Head Department of Applied Mathematics and Informatics, Perm State National Research University (15, Bukirev st., Perm, 614990, Russian Federation, e-mail: rusakov@ psu.ru).

Получено 02.07.2018 